\title{
Hervé Cavallera (éd.), Rudolf Steiner tra antroposofia e educazione
}

Lecce, Edizioni Pensa, Multimedia, 2006, 136 p.

Michel Ostenc

\section{OpenEdition}

\section{Journals}

Édition électronique

URL : http://journals.openedition.org/assr/22886

DOI : $10.4000 /$ assr.22886

ISSN : $1777-5825$

Éditeur

Éditions de l'EHESS

Édition imprimée

Date de publication : 31 décembre 2011

Pagination : 137

ISBN : 9782713223273

ISSN : 0335-5985

Référence électronique

Michel Ostenc, « Hervé Cavallera (éd.), Rudolf Steiner tra antroposofia e educazione », Archives de sciences sociales des religions [En ligne], 156 | octobre-décembre 2011, document 156-30, mis en ligne le 14 février 2012, consulté le 21 septembre 2020. URL : http://journals.openedition.org/assr/22886 ; DOI : https://doi.org/10.4000/assr.22886

Ce document a été généré automatiquement le 21 septembre 2020

(c) Archives de sciences sociales des religions 


\title{
Hervé Cavallera (éd.), Rudolf Steiner tra antroposofia e educazione
}

Lecce, Edizioni Pensa, Multimedia, 2006, 136 p.

\author{
Michel Ostenc
}

\section{RÉFÉRENCE}

Hervé Cavallera (éd.), Rudolf Steiner tra antroposofia e educazione, Lecce, Edizioni Pensa, Multimedia, 2006, $136 \mathrm{p}$.

1 La pensée et l'action éducative de Rudolf Steiner ont fait l'objet d'un colloque qui s'est tenu à Manduria (Italie) en 2004. La place de la connaissance de l'homme dans la formation des enseignants, celle de l'art et de la créativité dans les méthodes éducatives et l'importance de la liberté dans la pédagogie du penseur autrichien ont particulièrement retenu l'attention. Steiner avait travaillé aux archives de Weimar, de 1890 à 1897, avant de publier les écrits scientifiques de Goethe et d'écrire son premier livre important sur la Philosophie de la liberté (Die Philosophie der Freiheit, 1894). Il dirigea ensuite le Magasin for Literatur de Berlin. La pensée de Steiner était influencée par celle d'Eduard von Hartmann, l'inventeur de l'inconscient, et par des sources moins académiques comme les ouvrages d'Elena Petrovna Blavatsky qui l'avaient amené à la théosophie. Il se référait au christianisme, tout en ne situant le Christ que dans une vaste gamme de "Grands Initiés ", pour reprendre le titre d'Édouard Schuré. La théosophie se réclamait de Fichte pour critiquer le scientisme, la science n'étant pas pour elle un positivisme mais la compréhension intégrale de la personne humaine. Elle a pour principe l'intuition directe et prétend se distinguer du mysticisme par un appel à diverses sources de connaissances. La sensibilité devient ainsi le point de départ d'une sagesse éternelle qui entend rapprocher l'âme de la vérité. Steiner était imprégné de la lecture d'orientalistes allemands comme Dussen, un disciple de Kant et de Schopenhauer fortement influencé par la philosophie de l'Inde. Mais son orientalisme se heurtait à un ancrage occidental impliquant une sacralisation de l'individu, et il ne 
lui servait qu'à se réapproprier un héritage ésotérique fondé sur la méditation. Steiner se sépara des théosophes en leur reprochant un spiritisme grossier et il fonda la Société anthroposophique qui entendait étudier les dispositions naturelles de l'individu et ses capacités d'épanouissement. La « science de l'esprit » devait aider l'homme à conquérir des mondes nouveaux et à construire une liberté qui s'exprimait sous forme de besoins sensitifs, de sentiments et de représentations. Le penseur autrichien créa à Dornach en Suisse le théâtre Goetheanum où il donna des représentations de Faust et des cours d'art dramatique avec l'aide de son épouse Marie von Sivers. Ses activités s'étendirent aux domaines les plus divers et notamment à l'enseignement. Il dirigea à Stuttgart l'école Waldorf qui comptait plusieurs pédagogues dans son corps enseignant. Ses méthodes furent ensuite appliquées dans plusieurs établissements à l'étranger et sous son impulsion s'ouvrit même, en 1921, à Arlesheim un institut clinique préconisant une pédagogie curative fondée sur l'eurythmie. Ces activités pratiques étaient destinées à révéler les différences profondes séparant l'anthroposophie de l'illuminisme et de l'occultisme.

2 La théosophie prétend découvrir Dieu en étudiant la nature de l'homme et elle entend élever l'esprit jusqu'à son union avec la divinité ; mais le stade le plus élaboré de la pensée individuelle se situait pour Steiner dans les rapports de l'individu avec la société. Le penseur puisait chez Goethe l'idée de séparation des sens et de la pensée, la vérité s'ouvrant à l'observation plus qu'aux constructions de l'esprit. Le développement des facultés intellectuelles permet d'entrer en contact avec une réalité suprasensible et Sandra Christolini nous rappelle que chez Goethe la science et l'art n'excluaient pas la valeur de la représentation religieuse. Les principes stricts d'observation pratiqués par les sciences naturelles ne sont donc pas incompatibles avec la contemplation artistique et la chaleur de l'esprit peut concilier la rigueur scientifique avec une vision surnaturelle de l'univers. Anna Maria Colaci souligne pourtant la dualité de cette conception qui présente un homme toujours partagé entre l'attrait du monde et la répulsion qu'il lui inspire, l'anthroposophie ne parvenant pas à réaliser l'unité du moi et des choses. Cette incapacité à élaborer un système philosophique était au centre des critiques que lui adressait le néo-idéalisme italien. Certes, Giovanni Gentile se félicitait de découvrir chez Steiner une conception de la liberté qui était une célébration de l'individualité ; mais il la jugeait limitée à son caractère immanent et incapable de dépasser un empirisme critique.

3 Au-delà d'heureuses initiatives spiritualistes, l'individu anthroposophique finissait par n'être qu'un aspect particulier de la nature et les thèses de Steiner s'avéraient incapables de passer d'une théorie humaine à celle de la grâce divine. Vittorio Basile conclut que l'anthroposophie était incapable d'unir un idéalisme théorique et un réalisme pratique, ce qui représentait pour Gentile le fondement même de tout système philosophique. Steiner ne se contente d'ailleurs pas de s'éloigner du matérialisme: Hervé Cavallera prétend même qu'il prend ses distances avec le rationalisme, sa théorie de la connaissance restant fortement inspirée par les philosophies orientales. L'âme se défait difficilement des tentations du monde et le long cheminement de la connaissance la libère du corps pour qu'elle devienne esprit. L'homme prend ainsi conscience d'un destin qui reste imprécis, le devenir étant privé de sens et sa finalité vouée à la dissolution. Une connaissance authentique suppose un douloureux détachement du monde que Steiner assimilait à la Passion du Christ car elle condamne l'esprit à disparaître en se libérant de son asservissement au corps. L'arbre de la connaissance 
devient celui de la mort et non de la vie. Pour Cavallera, la dimension prophétique et le mysticisme de sa pensée conduisent Steiner à une sorte de religion laïque où la seule signification du monde est de permettre à l'homme d'accomplir son destin.

4 La nécessité d'aider son prochain est la raison d'être d'une pratique éducative qui trouve son fondement chez Steiner dans tous les aspects libératoires de l'enfant. Le principal objectif de la pédagogie consiste en un plein développement des forces physiques, psychiques et intellectuelles qui correspond à une connaissance anthroposophique de l'homme. Francesca Dello Preite est donc fondée à croire aux origines naturalistes d'une conception qui attribue à l'individu dès sa naissance toutes les parties constitutives de l'être. Pour que l'école soit celle de la vie, il lui faut des capacités d'adaptation aux prédispositions naturelles des élèves et à leur développement. La formation des écoles steinériennes se réclamait d'un idéalisme romantique que Sandra Christolini apparente au destin faustien de l'homme surmontant tous les obstacles pour aller toujours de l'avant. Ainsi, le corps et l'âme participent au langage pédagogique. Gabriele Armenise insiste au contraire sur l'importance de l'observation comme point de départ de tout processus de l'école Waldorf. L'éducation est un art capable d'accompagner l'individualité car Steiner concevait la liberté comme l'expression la plus authentique de la nature humaine; il faut aider son épanouissement en cultivant toutes les capacités de l'enfant dans les domaines de la volonté, de la sensation et de la pensée. L'élève se réalise lui-même à travers la subjectivité de ses sentiments. Toute éducation à la liberté individuelle pose l'éternel problème de l'initiation à la vie sociale et l'école Waldorf insistait sur les relations entre les personnes dans le contexte éducatif ; mais l'humanisme joue un rôle prioritaire et l'individu reste la source de toute morale. Les écoles de Steiner entendent préparer l'épanouissement personnel de l'élève et son insertion dans la vie sociale en alternant des activités artistiques, pratiques ou manuelles sollicitant l'esprit de créativité et des disciplines intellectuelles tournées vers l'abstraction. Elles multiplient les disciplines moins traditionnelles comme la musique, le dessin, la gymnastique ou le jardinage, avec la conviction que l'agilité du corps et des sens peut stimuler celle de la pensée.

5 Le développement de l'enfant obéit à trois cycles que Steiner déterminait en fonction de critères physiques liés à l'âge. Les parents jouent un rôle essentiel au premier stade de ce processus où la reproduction de modèles antérieurs s'effectue dans un milieu familial harmonieux et serein. L'enfant apprend ainsi à développer plusieurs types de sentiments et à respecter un premier ensemble de valeurs. Il structure ses démarches cognitives en fonction du langage. L'école réalise ensuite une grande partie de sa socialisation en établissant une hiérarchie fondée sur des classements qui relègue l'égalité de principe entre les élèves au niveau théorique. Steiner accordait une importante place aux méthodes actives qui laissaient à l'élève une grande part d'initiative dans la découverte du milieu; mais il justifiait l'éloignement du modèle parental au profit d'une attitude d'intériorité subjective par une série de métamorphoses naturelles. Anna Maria Colaci insiste sur la place de l'imagination dans la seconde étape de l'enseignement steinérien entre sept et quatorze ans, lorsque l'éducation sort de la phase ludique en suscitant la curiosité de l'enfant pour les phénomènes naturels et les activités humaines. À ce stade, l'élève conteste l'autorité au profit d'une force idéale représentée par le savoir de l'enseignant, l'œuvre d'un artiste ou une idéologie capable de capter son attention. L'intelligence n'apparaissant qu'avec la puberté, les différentes matières doivent être enseignées sous forme d'images et de 
symboles. L'enfant, mû par des sentiments, regarde la réalité avec des yeux d'artiste et l'art doit pénétrer toutes les disciplines en leur prêtant de nouvelles vertus. La géographie et les langues étrangères permettent à l'élève de s'ouvrir au monde, l'histoire servant d'exemple à un comportement idéal. La peinture, le chant et la récitation rendent le processus formatif moins ennuyeux et leur enseignement se déroule dans un environnement esthétique qui favorise l'esprit créatif. Les sensations artistiques précèdent la réflexion intellectuelle dans l'espoir d'éviter l'aridité épuisante d'une simple acquisition des connaissances et l'élément scientifique forme le jugement qui passe d'une logique hypothético-déductive à une pensée rationnelle. Mais l'accès à l'abstraction passait pour Steiner par une « science spirituelle » et le développement de l'esprit critique ne devait pas être prématuré. La formation de la liberté de jugement connaît ainsi plusieurs étapes: une méditation esthétique qui permet à l'enfant d'accéder au stade de l'imagination, l'inspiration qui lui offre un premier contact avec l'esprit et l'intuition qui lui ouvre les portes de la connaissance.

Le sacerdoce du maître de l'école de Waldorf était mis au service d'une vraie connaissance de l'être humain et son autorité exigeait des rapports de confiance qu'il pouvait cultiver en suivant ses élèves pendant tout un cycle d'enseignement. L'acquisition du savoir reste progressive au terme d'un parcours accompli ensemble par le maître et ses élèves. Un même souci d'unité incite les écoles de Steiner à consacrer deux heures par jour pendant des périodes de trois à cinq semaines à une matière afin d'en faciliter la compréhension par l'absence de toute fragmentation dans son étude. L'enseignant reste libre de ses initiatives en fonction du développement de l'enfant et le plan d'études idéal doit s'inspirer d'une nature humaine en devenir.

7 Steiner considérait les sensations empiriques de l'enfant comme les premières manifestations d'un sens artistique qui doit contribuer à un développement harmonieux du processus éducatif. On conviendra avec lui que l'éducation n'est faite qu'en partie de transmission, la continuité n'étant pas pour elle une fin en soi. En outre, nul ne sait au juste comment se forme la créativité artistique et on ne peut éluder à ce propos la question de l'inégalité des dons innés, ni celle de l'inégalité des chances. Si bien que la recherche de la créativité à tout prix peut sombrer dans des pratiques dénuées de capacités formatrices. Cavallera place ainsi l'intuition au centre de la pensée de Steiner, les sensations, les sentiments et l'imagination devenant les sources authentiques de l'épanouissement de la personnalité. L'intuition n'est pas la raison et l'esprit critique suppose une formation intellectuelle préalable dont Steiner se méfie par crainte qu'elle ne fasse obstacle à la liberté de jugement. En fait, il inverse la tendance en privilégiant des critères subjectifs comme l'inspiration au détriment de la pensée rationnelle. Cet anti-intellectualisme se veut compensé par les séductions d'un humanisme plein de liberté spontanée qui condamne sans démagogie l'autoritarisme, tout en revendiquant la nécessité de l'autorité. Cet humanisme n'en reste pas moins mystique dans la mesure où la fin de l'humanité lui apparaît comme une dissolution dans le cosmos. Steiner s'efforce vainement de concilier les impératifs d'une étude anthropologique de la nature humaine et les exigences intellectuelles de la pédagogie. 